\title{
Metrics in the sphere of a $\mathrm{C}^{*}$-module*
}

\author{
Esteban Andruchow and Alejandro Varela
}

Instituto de Ciencias, Univ. Nac. de Gral. Sarmiento, Argentina

\begin{abstract}
Given a unital $C^{*}$-algebra $\mathcal{A}$ and a right $C^{*}$-module $\mathcal{X}$ over $\mathcal{A}$, we consider the problem of finding short smooth curves in the sphere $\mathcal{S}_{\mathcal{X}}=\{x \in \mathcal{X}:<x, x>=1\}$. Curves in $\mathcal{S}_{\mathcal{X}}$ are measured considering the Finsler metric which consists of the norm of $\mathcal{X}$ at each tangent space of $\mathcal{S}_{\mathcal{X}}$. The initial value problem is solved, for the case when $\mathcal{A}$ is a von Neumann algebra and $\mathcal{X}$ is selfdual: for any element $x_{0} \in \mathcal{S}_{\mathcal{X}}$ and any tangent vector $v$ at $x_{0}$, there exists a curve $\gamma(t)=e^{t Z}\left(x_{0}\right)$, $Z \in \mathcal{L}_{\mathcal{A}}(\mathcal{X}), Z^{*}=-Z$ and $\|Z\| \leq \pi$, such that $\gamma(0)=x_{0}$ and $\dot{\gamma}(0)=v$, which is minimizing along its path for $t \in[0,1]$. The existence of such $Z$ is linked to the extension problem of selfadjoint operators. Such minimal curves need not be unique. Also we consider the boundary value problem: given $x_{0}, x_{1} \in \mathcal{S}_{\mathcal{X}}$, find a curve of minimal length which joins them. We give several partial answers to this question. For instance, let us denoteb
\end{abstract}

Keywords: $\mathrm{C}^{*}$-modules, spheres, geodesics.

\section{Introduction}

The sphere $\mathcal{S}_{\mathcal{X}}$ of a right Hilbert $C^{*}$-module $\mathcal{X}$ over a unital $C^{*}$-algebra $\mathcal{A}$, which consists of the elements $x \in \mathcal{X}$ such that $\langle x, x\rangle=1$, is a $\mathrm{C}^{\infty}$ submanifold of the (Banach space) $\mathcal{X}$. Its basic topological and differentiable aspects were considered in [2]. In this paper we consider the geometric problem of finding short smooth curves in $\mathcal{S}_{\mathcal{X}}$. To measure the length of a smooth curve we endow each tangent space (which we describe below, and is a complemented real Banach subspace of $\mathcal{X}$ ), with the norm of $\mathcal{X}$. Therefore the length of a curve $\gamma(t) \in \mathcal{S}_{\mathcal{X}}, t \in[a, b]$ is measured by

$$
\text { length }(\gamma)=\int_{a}^{b}\|\dot{\gamma}(t)\| d t,
$$

where \|\| denotes the norm of $\mathcal{X}$. We refer the reader to [11] for basic facts on $C^{*}$-modules. As is usual notation, let $\mathcal{L}_{\mathcal{A}}(\mathcal{X})$ be the $C^{*}$-algebra of adjointable linear operators acting on $\mathcal{X}$. If $y, z \in \mathcal{X}$, let $y \otimes z \in \mathcal{L}_{\mathcal{A}}(\mathcal{X})$ be the operator $y \otimes z(x)=y<z, x>$. For example, it is easy to see that if $x \in \mathcal{S}_{\mathcal{X}}$, then $x \otimes x$ is a selfadjoint projection, which we shall denote by $e_{x}$. Let $\mathcal{U}(\mathcal{X})$ be the unitary group of $\mathcal{L}_{\mathcal{A}}(\mathcal{X})$. Perhaps the main feature in the geometry of $\mathcal{S}_{\mathcal{X}}$ (as with classical spheres) is the natural action of $\mathcal{U}(\mathcal{X})$ on $\mathcal{S}_{\mathcal{X}}$ :

$$
U \cdot x=U(x), \quad U \in \mathcal{U}(\mathcal{X}), x \in \mathcal{S}_{\mathcal{X}} .
$$

*2000 Mathematics Subject Classification: 46L08, 53C22, 58B20. 
In [2] it was shown that if $x_{0}, x_{1} \in \mathcal{S}_{\mathcal{X}}$ verify $\left\|x_{0}-x_{1}\right\|<1 / 2$, then $x_{0}$ and $x_{1}$ are conjugate by this action, moreover, one can find a unitary operator $U_{\left(x_{0}, x_{1}\right)}$, which is a $\mathrm{C}^{\infty}$ function in $\left(x_{0}, x_{1}\right)$ such that $U_{\left(x_{0}, x_{1}\right)}\left(x_{0}\right)=x_{1}$. In particular the action is locally transitive. It is globally transitive in some cases (e.g. if $\mathcal{X}$ is selfdual [15] and $\mathcal{A}$ is a finite von Neumann algebra). In general, $\mathcal{S}_{\mathcal{X}}$ has many components: take for instance $\mathcal{X}=\mathcal{B}(\mathcal{H})$ with the inner product $\langle X, Y\rangle=X^{*} Y$, then the sphere is the set of isometries of $\mathcal{H}$, whose connected components are parametrized by the codimension of the range.

The existence of local cross sections for the action (namely, the unitaries $U_{\left(x_{0}, x_{1}\right)}$ ), implies that for any fixed $x_{0} \in \mathcal{S}_{\mathcal{X}}$, the map

$$
\pi_{x_{0}}: \mathcal{U}(\mathcal{X}) \rightarrow \mathcal{S}_{\mathcal{X}}, \quad \pi_{x_{0}}(U)=U\left(x_{0}\right)
$$

is a locally trivial fibre bundle and a $\mathrm{C}^{\infty}$ submersion. It follows that any smooth curve $\gamma(t) \in \mathcal{S}_{\mathcal{X}}$ can be lifted to a smooth curve $\mu(t) \in \mathcal{U}(\mathcal{X})$, and therefore represented $\gamma(t)=\mu(t) \cdot x_{0}$ for some $x_{0} \in \mathcal{S}_{\mathcal{X}}$. This enables one to compute the tangent spaces of $\mathcal{S}_{\mathcal{X}}$ :

$$
\left(T \mathcal{S}_{\mathcal{X}}\right)_{x_{0}}=\left\{A\left(x_{0}\right): A \in \mathcal{L}_{\mathcal{A}}(\mathcal{X}), A^{*}=-A\right\}
$$

Clearly these elements are also characterized by the condition

$$
\left(T \mathcal{S}_{\mathcal{X}}\right)_{x_{0}}=\left\{v \in \mathcal{X}:<v, x_{0}>+<x_{0}, v>=0\right\}
$$

It is natural to ask whether one can find curves of the form

$$
\gamma(t)=e^{t Z}\left(x_{0}\right), \quad t \in[0,1], Z^{*}=-Z,
$$

which have minimal length joining their endpoints, or more strictly, which have minimal length along their paths.

There are two main problems.

1. The initial value problem: for any tangent vector $v \in\left(T \mathcal{S}_{\mathcal{X}}\right)_{x_{0}}$ find a curve $\gamma$ as above (in particular $\left.\gamma(0)=x_{0}\right)$, with $\dot{\gamma}(0)=v$, such that $\gamma$ has minimal length.

2. The boundary value problem: given $x_{0}, x_{1}$ in the same component of $\mathcal{S}_{\mathcal{X}}$, find a minimal curve $\gamma$ as above, which joins $x_{0}$ and $x_{1}$.

In this paper we solve the initial value problem: we show that if $\mathcal{A}$ is a von Neumann algebra and $\mathcal{X}$ is a right $C^{*}$-module, which is selfdual [15], then for any $x_{0} \in \mathcal{S}_{\mathcal{X}}$ and any tangent vector $v \in\left(T \mathcal{S}_{\mathcal{X}}\right)_{x_{0}}$ with $\|v\| \leq \pi$ there exists a curve $\gamma(t)=e^{t Z}\left(x_{0}\right)$ with $\gamma(0)=x_{0}$ and $\dot{\gamma}(0)=v$, which has minimal length along its path for $t \in[0,1]$. The antihermitic operator $Z$ implementing this geodesic is the solution of the extension problem by M.G. Krein [10], in the context of von Neumann algebras (see [6]), as it will be shown in the next section. We call such $Z$ minimal lifts, following [7].

We also consider the boundary value problem. We prove that if $\left\langle x_{0}, x_{1}\right\rangle$ is a scalar multiple of the identity, then $x_{0}$ and $x_{1}$ can be joined by a minimizing geodesic (Proposition (4.1)). Another case in which there exists a short geodesic joining $x_{0}$ and $x_{1}$ occurs when the (non empty) set $\left\{\|Z\|: Z^{*}=-Z, e^{Z}\left(x_{0}\right)=x_{1}\right\}$ has a minimum (Theorem (4.3)). As a consequence, we obtain that if $f_{0}(\mathcal{X})$ is finite dimensional $\left(f_{0}=I-e_{x_{0}}\right)$, then there exists such a geodesic. In section 5 we introduce a metric in $\mathcal{S}_{\mathcal{X}}$, by means of the states of $\mathcal{A}$, which induce Hilbert space representations of the sphere $\mathcal{S}_{\mathcal{X}}$. We compare this metric with the Finsler metric. For example, it is shown that they coincide whenever there exist minimal lifts (Theorem (5.4)). 


\section{Extension problem in von Neumann algebras}

A simplified version of the extension problem ([10], [14], [6]) could be stated as follows: given a closed subspace $\mathcal{L}$ of a Hilbert space $\mathcal{H}$ and a bounded symmetric operator $A_{0}: \mathcal{L} \rightarrow \mathcal{H}$, find a selfadjoint extension $A: \mathcal{H} \rightarrow \mathcal{H}$ with $\|A\|=\left\|A_{0}\right\|$. This problem was solved, and all solution parametrized. We remark that extensions can, but in general need not, be unique. See for example [6] or [14] for explicit parametrizations. M.G. Krein [10] showed that there exist a minimal and a maximal solution (in terms of the usual order of selfadjoint operators), and that all solution lie in between. For our purposes, we need the additional requierement that if $P=P_{\mathcal{L}}$ (=the orthogonal projection onto $\mathcal{L}$ ) and $A_{0}$ lie in a von Neumann algebra $\mathcal{B}$, then there exists a solution of the extension problem in $\mathcal{B}$. By this we mean the following result, which is a consequence of the parametrization of solutions given by Davis, Kahan and Wi

Lemma 2.1 Let $A$ be a selfadjoint element and $P$ a selfadjoint projection in a von Neumann algebra $\mathcal{B}$. Then there exists a selfadjoint element $Z$ in $\mathcal{B}$ such that $Z P=A P$ and $\|Z\|=\|A P\|$.

Proof. Let $A$ and $P \in \mathcal{B}$ be as above. Choose a representation of the von Neumann algebra $\mathcal{B}$ in $\mathcal{B}(\mathcal{H})$ with $\mathcal{H}$ a Hilbert space. Let us consider the following selfadjoint $2 \times 2$ block operators in terms of $P$ and $(I-P)$ :

$$
Z_{X}=\left(\begin{array}{cc}
P A P & (I-P) A P \\
P A(I-P) & X
\end{array}\right)
$$

where $X$ is a selfadjoint operator in $\mathcal{B}((I-P) \mathcal{H})$. These $Z_{X} \in \mathcal{B}(\mathcal{H})$ satisfy $Z_{X} P=A P$ and $\left\|Z_{X}\right\| \geq\|A P\|$.

As it was mentioned at the begining of this section, several authors dealt with the problem of minimizing the norm of $Z_{X}$. Theorem 1 in [14], for example, proves that in our context there exists an $X_{0} \in \mathcal{B}((I-P) \mathcal{H})$ such that $\left\|Z_{X_{0}}\right\|=\|P A\|$ and $X_{0}$ is the weak limit of the following elements of $\mathcal{B}:-c_{n}(I-P)\left(I-d_{n} P A P A P\right)^{-1} P A P A(I-P)$ (where $\left\{c_{n}\right\}$ and $\left\{d_{n}\right\}$ are sequences of real numbers). Therefore this $X_{0}$ belongs to $\mathcal{B}$ and then $Z_{X_{0}}$ belongs to $\mathcal{B}$, and verifies $\left\|Z_{X_{0}}\right\|=\|P A\|$.

We state now a consequence of the result above, in the context of the modular spheres. Let $x_{0} \in \mathcal{S}_{\mathcal{X}}$, and $v \in\left(T \mathcal{S}_{\mathcal{X}}\right)_{x_{0}}$. We call an antihermitic operator $Z \in \mathcal{L}_{a}(\mathcal{X})$ a minimal lift of $v$ if $Z\left(x_{0}\right)=v$ and $\|Z\|=\|v\|$.

Corollary 2.2 Let $x_{0} \in \mathcal{S}_{\mathcal{X}}$, with $\mathcal{X}$ a selfdual module over the von Neumann algebra $\mathcal{A}$, and $v \in\left(T \mathcal{S}_{x}\right)_{x_{0}}$. Then there exists a minimal lift $Z$ of $v$.

Proof. In this case, $\mathcal{L}_{\mathcal{A}}(\mathcal{X})$ is a von Neumann algebra [15]. Since $v \in\left(T \mathcal{S}_{x}\right)_{x_{0}}$, there exists $A \in \mathcal{L}_{\mathcal{A}}(\mathcal{X})$ such that $-A=A^{*}$ and $A\left(x_{0}\right)=v$. Note that this implies that $A\left(x_{0} \otimes x_{0}\right)=v \otimes x_{0}$. Moreover, the operator $v \otimes x_{0}$ has norm equal to the norm of $v$. Indeed, clearly $\left\|v \otimes x_{0}\right\| \leq$ $\|v\|\left\|x_{0}\right\|=\|v\|$ because $\left\|x_{0}\right\|=1$, and $\left\|v \otimes x_{0}\right\| \geq\left\|v \otimes x_{0}\left(x_{0}\right)\right\|=\|v\|$. Since $e_{x_{0}}=x_{0} \otimes x_{0}$ is a selfadjoint projection in $\mathcal{L}_{\mathcal{A}}(\mathcal{X})$, by the above lemma there exists $Z \in \mathcal{L}_{\mathcal{A}}(\mathcal{X})$ such that $Z^{*}=-Z$, $Z e_{x_{0}}=A e_{x_{0}}$ and $\|Z\|=\left\|A e_{x_{0}}\right\|$. In other words, $Z\left(x_{0}\right)=Z e_{x_{0}}\left(x_{0}\right)=A e_{x_{0}}\left(x_{0}\right)=A\left(x_{0}\right)=v$, and $\|Z\|=\|v\|$.

\section{The initial value problem}

Let us state our main result. 
Theorem 3.1 Let $x_{0} \in \mathcal{S}_{\mathcal{X}}$ and $v \in\left(T \mathcal{S}_{\mathcal{X}}\right)_{x_{0}}$ with $\|v\| \leq \pi$. Let $Z$ be a minimal lift of $v$, i.e.

$$
Z^{*}=-Z, Z\left(x_{0}\right)=v \text { and }\|Z\|=\|v\| .
$$

Then the curve $\nu(t)=e^{t Z}\left(x_{0}\right), t \in[0,1]$ which verifies $\nu(0)=x_{0}$ and $\dot{\nu}(0)=v$, has minimal length along its path among smooth curves in $\mathcal{S}_{\mathcal{X}}$.

Proof. Given a positive element $A$ of a $C^{*}$-algebra, there exists a faithful representation of the algebra (for instance, the universal representation) and a unit vector $\xi$ in the Hilbert space $\mathcal{H}$ of this representation, such that $A \xi=\|A\| \xi$ (here we identify $A$ with its image under the representation). Let us call such a vector $\xi$ a norming eigenvector for $A$. Let us apply this folklore fact to the positive operator $-e Z^{2} e$, where $e=e_{x_{0}}$. Let $\xi$ be a (unit) norming eigenvector for $-e Z^{2} e$. Again we identify the operators with their images under this representation, and regard them as operators in this Hilbert space. Clearly $\xi$ lies in the range of $e$. We claim that $\xi$ is a norming eigenvector for $-Z^{2}$ as well. Indeed,

$$
-Z^{2} \xi=-Z^{2} e \xi=-e Z^{2} P \xi-(I-e) Z^{2} P \xi=\left\|e Z^{2} e\right\| \xi+\xi_{1},
$$

where $\xi_{1}=-(I-e) Z^{2} e \xi$ is orthogonal to $\xi$. Note that

$$
\left\|e Z^{2} e\right\|=\|Z e\|^{2}=\|Z\|^{2}=\left\|Z^{2}\right\|
$$

Then

$$
\left\|Z^{2}\right\|^{2} \geq\left\|Z^{2} \xi\right\|^{2}=\left\|e Z^{2} e\right\|^{2}+\left\|\xi_{1}\right\|^{2}=\left\|Z^{2}\right\|^{2}+\left\|\xi_{1}\right\|^{2} .
$$

It follows that $\xi_{1}=0$ and our claim is proved. Consider the curve $\hat{\nu}(t)=e^{t Z}(\xi)$. Clearly $\|\nu \hat{(t)}\|=1$, i.e. $\hat{\nu}(t)$ is a curve in the unit sphere $S_{\mathcal{H}}$ of the Hilbert space $\mathcal{H}$. Let us prove that it is a minimizing geodesic of this Riemann-Hilbert manifold. Indeed,

$$
\ddot{\hat{\nu}}(t)=e^{t Z} Z^{2} \xi=-\|Z\|^{2} e^{t Z} \xi=-\|Z\|^{2} \hat{\nu}(t) .
$$

That is, $\hat{\nu}$ satisfies the differential equation of the geodesics of the sphere $S_{\mathcal{H}}$. Moreover, the length of $\hat{\nu}$ is

$$
\text { length }(\hat{\nu})=\int_{0}^{1}\|\dot{\hat{\nu}}(t)\| d t=\|Z \xi\| \leq \pi .
$$

It follows that $\hat{\nu}$ is a minimizing geodesic of the unit sphere. Note also that

$$
\|Z \xi\|^{2}=<Z \xi, Z \xi>=<-Z^{2} \xi, \xi>=\left\|Z^{2}\right\|=\|Z\|^{2} .
$$

Clearly, if $\left[t_{0}, t_{1}\right] \subset[0,1]$, the length of $\hat{\nu}$ restricted to $\left[t_{0}, t_{1}\right]$ (or shortly $\left.\left.\hat{\nu}\right|_{\left[t_{0}, t_{1}\right]}\right)$ is $\left(t_{1}-t_{0}\right)\|Z\|$. On the other hand,

$$
\operatorname{length}\left(\left.\nu\right|_{\left[t_{0}, t_{1}\right]}\right)=\int_{t_{0}}^{t_{1}}\|\dot{\nu}\| d t=\left(t_{1}-t_{0}\right)\left\|Z\left(x_{0}\right)\right\|=\left(t_{1}-t_{0}\right)\|Z\| .
$$

It follows that length $(\hat{\nu})=$ length $(\nu)$ on any subinterval of $[0,1]$.

Suppose now that $\gamma:[a, b] \rightarrow \mathcal{S}_{\mathcal{X}}$ is a smooth curve joining $\nu\left(t_{0}\right)$ and $\nu\left(t_{1}\right)$. Consider the curve $\hat{\gamma}(t):=\gamma(t) \otimes x_{0}(\xi)$. Note that $\hat{\gamma}$ is also a curve in the unit sphere of $\mathcal{H}$ :

$<\hat{\gamma}(t), \hat{\gamma}(t)>_{\mathcal{H}}=<\left(\gamma(t) \otimes x_{0}\right)^{*}\left(\gamma(t) \otimes x_{0}\right) \xi, \xi>_{\mathcal{H}}=<\left(x_{0} \otimes \gamma(t)\right)(\gamma(t)) \otimes x_{0} \xi, \xi>_{\mathcal{H}}=<e \xi, \xi>_{\mathcal{H}}=1$.

Moreover,

$$
\|\dot{\hat{\gamma}}(t)\|=\left\|\left(\dot{\gamma}(t) \otimes x_{0}\right) \xi\right\| \geq\left\|\dot{\gamma}(t) \otimes x_{0}\right\|=\|\dot{\gamma}(t)\| .
$$


This implies that length $(\gamma) \leq \operatorname{length}(\hat{\gamma})$. Finally, let us show that $\left.\hat{\nu}\right|_{\left[t_{0}, t_{1}\right]}$ and $\hat{\gamma}$ join the same endpoints of $\mathcal{S}_{\mathcal{H}}$ :

$\hat{\nu}\left(t_{0}\right)=e^{t_{0} Z} \xi=e^{t_{0} Z} e \xi=e^{t_{0} Z}\left(x_{0} \otimes x_{0}\right) \xi=\left(e^{t_{0} Z}\left(x_{0}\right) \otimes x_{0}\right) \xi=\left(\nu\left(t_{0}\right) \otimes x_{0}\right) \xi=\left(\gamma\left(t_{0}\right) \otimes x_{0}\right) \xi=\hat{\gamma}\left(t_{0}\right)$,

and similarly for $t_{1}$. By the minimality of $\hat{\nu}$, it follows that length $\left(\left.\hat{\nu}\right|_{\left[t_{0}, t_{1}\right]}\right) \leq \operatorname{length}(\hat{\gamma})$. Therefore

$$
\operatorname{length}\left(\left.\nu\right|_{\left[t_{0}, t_{1}\right]}\right)=\operatorname{length}\left(\left.\nu\right|_{\left[t_{0}, t_{1}\right]}\right) \leq \text { length }(\hat{\gamma}) \leq \operatorname{lenght}(\gamma),
$$

which completes the proof.

Corollary 3.2 If $\mathcal{A}$ is a von Neumann algebra and $\mathcal{X}$ is a selfdual module, then for any element $x_{0} \in \mathcal{S}_{\mathcal{X}}$ and tangent vector $v \in\left(T \mathcal{S}_{\mathcal{X}}\right)_{x_{0}}$ with $\|v\| \leq \pi$, there exists a geodesic $\delta$ with $\delta(0)=x_{0}$, $\dot{\delta}(0)=v$, such that $\delta$ is minimizing along its path for $t \in[0,1]$.

Proof. In this case, minimal lifts exist for any tangent vector $v$.

\section{Geodesics joining given endpoints}

The problem of finding minimizing geodesics given any pair of points (in the same component) of the sphere $\mathcal{S}_{\mathcal{X}}$ is more difficult. It is related to the analogous problem for abstract homogeneous spaces [8]. In this section we find solutions in certain cases. These results work for arbitrary $C^{*}$-algebras and modules.

Proposition 4.1 Let $x_{0}, x_{1} \in \mathcal{S}_{\mathcal{X}}$ with $\left\langle x_{0}, x_{1}\right\rangle=\alpha .1$, for $\alpha \in \mathbb{C}$. Then there exists a smooth curve in $\mathcal{S}_{\mathcal{X}}$ with minimal length along its path, which joins $x_{0}$ and $x_{1}$.

Proof. Note that since $\left\|<x_{0}, x_{1}>\right\| \leq\left\|x_{0}\right\|\left\|x_{1}\right\|=1$, it follows that $|\alpha| \leq 1$. If $|\alpha|=1$, then $\alpha=e^{i r}$ with $|r| \leq \pi$. In this case clearly $x_{1}=\alpha x_{0}$. Indeed,

$$
<x_{1}-\alpha x_{0}, x_{1}-\alpha x_{0}>=<x_{1}, x_{1}>-<x_{1}, \alpha x_{0}>-<\alpha x_{0}, x_{1}>+<\alpha x_{0}, \alpha x_{0}>=0 \text {. }
$$

Put $\gamma(t)=e^{i r t} x_{0}$. Apparently $\gamma$ is minimizing along its path (for instance, $\|r e\|=r$, i.e. the operator $r I$ is a minimal lift).

If $|\alpha|<1$, let $\beta \in \mathbb{C}$ be such that $|\alpha|^{2}+|\beta|^{2}=1$ (note that $\beta \neq 0$ ), and consider $y=$ $\alpha \beta^{-1} x_{0}-\beta^{-1} x_{1}$. Then clearly

$$
<x_{0}, y>=\alpha \beta^{-1} 1-\beta^{-1}<x_{0}, x_{1}>=0,
$$

and

$$
<y, y>=\frac{|\alpha|^{2}}{|\beta|^{2}}-\frac{\bar{\alpha}}{|\beta|^{2}}<x_{0}, x_{1}>-\frac{\alpha}{|\beta|^{2}}<x_{1}, x_{0}>+\frac{1}{|\beta|^{2}}=1 .
$$

in other words, $x_{1}=\alpha x_{0}+\beta y$ with $y \in \mathcal{S}_{\mathcal{X}}$. That is, $x_{1}$ lies in the complex plane generated by two orthogonal elements $x_{0}$ and $y$ of $\mathcal{S}_{\mathcal{X}}$. The situation resembles what happens in a classic finite dimensional sphere, and the proof follows as in that case. Namely, let $(\alpha(t), \beta(t))$ be a minimal geodesic of the sphere $\mathcal{S}_{\mathbb{C}^{2}}$ of $\mathbb{C}^{2}$, joining $(1,0)($ at $t=0)$ and $(\alpha, \beta)$ (at $\left.t=1\right)$. Consider the curve $\gamma(t)=\alpha(t) x_{0}+\beta(t) y$. Clearly it is a smooth curve with $\gamma(0)=x_{0}$ and $\gamma(1)=x_{1}$, which lies in $\mathcal{S}_{\mathcal{X}}$ :

$$
<\gamma(t), \gamma(t)>=|\alpha(t)|^{2}+|\beta(t)|^{2}=1
$$

Moreover, it has constant speed equal to

$$
\|\dot{\gamma}(t)\|^{2}=\left\|<\dot{\alpha}(t) x_{0}+\dot{\beta}(t) x_{1}, \dot{\alpha}(t) x_{0}+\dot{\beta}(t) x_{1}>\right\|^{2}=|\dot{\alpha}(t)|^{2}+|\dot{\beta}(t)|^{2}=|\dot{\alpha}(0)|^{2}+|\dot{\beta}(0)|^{2}=\|\dot{\gamma}(0)\|^{2} .
$$


We claim that it is minimizing along its path. Let $\varphi$ be a state in $\mathcal{A}$. Then the form

$$
[x, y]_{\varphi}:=\varphi(<x, y>), \quad x, y \in \mathcal{X}
$$

is positive semidefinite in $\mathcal{X}$. Let $\mathcal{H}_{\varphi}$ be the completion of $\left(\mathcal{X} / \mathcal{Z},[,]_{\varphi}\right)$, where $\mathcal{Z}=\{z \in \mathcal{X}$ : $\left.[z, z]_{\varphi}=0\right\}$. Denote by $\bar{x}$ be the class of $x \in \mathcal{X}$ in $\mathcal{X} / \mathcal{Z} \subset \mathcal{H}_{\varphi}$. In other words, $\bar{x}$ is the element $x$ regarded as a vector in the Hilbert space $\mathcal{H}_{\varphi}$. Note that the elements of $\mathcal{S}_{\mathcal{X}}$ induce elements in the unit sphere of $\mathcal{H}_{\varphi}$ : clearly $\left.[\bar{x}, \bar{x}]_{\varphi}=\varphi(<x, x\rangle\right)=1$

The geodesic $(\alpha(t), \beta(t))$ of $\mathcal{S}_{\mathbb{C}^{2}}$ satisfies the Euler equation of the sphere:

$$
(\ddot{\alpha}(t), \ddot{\beta}(t))=-\kappa^{2}(\alpha(t), \beta(t)) .
$$

It follows that $\bar{\gamma}$ satisfies the differential equation

$$
\ddot{\bar{\gamma}}(t)=-\kappa^{2} \bar{\gamma}(t)
$$

in the sphere $\mathcal{S}_{\mathcal{H}_{\varphi}}$ of $\mathcal{H}_{\varphi}$. Moreover, the length of $\bar{\gamma}$ restricted to the interval $\left[t_{1}, t_{2}\right] \subset[0,1]$, is given by

$$
\begin{gathered}
\int_{t_{0}}^{t_{1}}[\dot{\bar{\gamma}}(t), \dot{\bar{\gamma}}(t)]^{1 / 2} d t=\int_{t_{0}}^{t_{1}} \varphi\left(<\dot{\alpha}(t) x_{0}+\dot{\beta}(t) y, \dot{\alpha}(t) x_{0}+\dot{\beta}(t) y>\right)^{1 / 2} d t \\
=\int_{t_{0}}^{t_{1}} \varphi\left(|\dot{\alpha}(t)|^{2} \cdot 1+|\dot{\beta}(t)|^{2} .1\right)^{1 / 2} d t=\left(t_{1}-t_{0}\right)\|\dot{\gamma}(0)\| .
\end{gathered}
$$

It follows that $\bar{\gamma}$ is minimizing along its path in $\mathcal{S}_{\mathcal{H}_{\varphi}}$, and

$$
\text { length }(\bar{\gamma})=\text { length }(\gamma) \text {. }
$$

Let $\nu(t), t \in[0,1]$ be another smooth curve in $\mathcal{S}_{\mathcal{X}}$ joining $\nu(0)=\gamma\left(t_{0}\right)$ and $\nu(1)=\gamma\left(t_{1}\right)$. Then $\bar{\nu}$ is a smooth curve in $\mathcal{S}_{\mathcal{H}_{\varphi}}$, and the inequality

$$
[\dot{\bar{\nu}}, \dot{\bar{\nu}}]_{\varphi}=\varphi(<\dot{\nu}, \dot{\nu}>) \leq\|<\dot{\nu}, \dot{\nu}>\|
$$

implies that

$$
\text { length }(\nu) \geq \text { length }(\bar{\nu}) .
$$

It follows that $\nu$ is not shorter than $\left.\gamma\right|_{\left[t_{0}, t_{1}\right]}$.

If $x_{0}, x_{1} \in \mathcal{S}_{\mathcal{X}}$ satisfy that $\left\|x_{0}-x_{1}\right\|<1 / 2$, then they are conjugate by the action of $\mathcal{U}(\mathcal{X})$ (see [2]). Let us state the following result, estimating the distance between the identity and the unitary operator performing this conjugacy.

Lemma 4.2 Let $x_{0}, x_{1} \in \mathcal{S}_{\mathcal{X}}$ with $\left\|x_{0}-x_{1}\right\|<1 / 2$. Then there exists a unitary $U \in \mathcal{U}(\mathcal{X})$ such that $U\left(x_{0}\right)=x_{1}$ with $\|U-I\|<3 / 2$.

Proof. First we transcribe the construction of the unitary $U$ given in [2]. Let $e_{0}=e_{x_{0}}$ and $e_{1}=e_{x_{1}}$. Since $\left\|x_{0}-x_{1}\right\|<1 / 2$, it follows that

$$
\left\|e_{0}-e_{1}\right\| \leq\left\|e_{0}-x_{0} \otimes x_{1}\right\|+\left\|x_{0} \otimes x_{1}-e_{1}\right\|=\left\|x_{0} \otimes\left(x_{0}-x_{1}\right)\right\|+\left\|\left(x_{1}-x_{0}\right) \otimes x_{1}\right\| .
$$

Note that $\left\|x_{0} \otimes\left(x_{0}-x_{1}\right)\right\| \leq\left\|x_{0}-x_{1}\right\|$ (in fact equality holds because $x_{0} \in \mathcal{S}_{\mathcal{X}}$ ), and analogously for the other term. Therefore $\left\|e_{0}-e_{1}\right\|<1$. It is a standard fact that two such projections are unitarily equivalent, morever, the unitary $V$ such that $V e_{0} V^{*}=e_{1}$ can be chosen $V=e^{Y}$ with 
$Y \in \mathcal{L}_{\mathcal{A}}(\mathcal{X})$ such that $Y^{*}=-Y$ and $\|Y\|<\pi / 2$ (moreover, $Y$ is codiagonal in terms of $e_{0}$ and $\sin \|Y\|=\left\|e_{0}-e_{1}\right\|$, see for instance [3], page 151). Therefore

$$
\|I-V\|=r(I-V)=\operatorname{supp}\left\{\left|1-e^{\omega}\right|: \omega \in \operatorname{sp}(Y)\right\}<\sqrt{2},
$$

because $|\omega| \leq\|Y\|<\pi / 2$ (here $s p$ and $r$ stand for the spectrum and the spectral radius, respectively). Consider

$$
U=x_{1} \otimes x_{0}+V\left(I-e_{0}\right) .
$$

This unitary verifies that $U\left(x_{0}\right)=x_{1}$, and moreover,

$$
\|I-U\|=\left\|e_{1}-x_{1} \otimes x_{0}+\left(I-e_{1}\right)-V\left(I-e_{0}\right)\right\| .
$$

Since $V\left(I-e_{0}\right) V^{*}=e_{1}$, it follows that the operators $e_{1}-x_{1} \otimes x_{0}$ and $\left(I-e_{1}\right)-V\left(I-e_{0}\right)$ have orthogonal ranges (in any Hilbert space representation for $\mathcal{L}_{\mathcal{A}}(\mathcal{X})$ ). Indeed, the range of $e_{1}-x_{1} \otimes x_{0}=e_{1}\left(I-x_{1} \otimes x_{0}\right)$ is contained in the range of $e_{1}$, and the range of $\left(I-e_{1}\right)-V\left(I-e_{0}\right)=$ $\left(I-e_{1}\right)-\left(I-e_{1}\right) V^{*}$ is contained in its orthogonal complement. Thus

$$
\|I-U\| \leq \sqrt{\left\|e_{1}-x_{1} \otimes x_{0}\right\|^{2}+\left\|I-e_{0}-V\left(I-e_{0}\right)\right\|^{2}} .
$$

Note that $\left\|e_{1}-x_{1} \otimes x_{0}\right\|=\left\|x_{1} \otimes\left(x_{1}-x_{0}\right)\right\|=\left\|x_{1}-x_{0}\right\|<1 / 2$ and

$$
\left\|I-e_{0}-V\left(I-e_{0}\right)\right\|=\left\|\left(I-e_{0}\right)(I-V)\right\| \leq\|I-V\| \leq \sqrt{2} .
$$

Then

$$
\|I-U\|<3 / 2
$$

In particular, by a standard argument involving the continuous functional calculus in the $C^{*}$-algebra $\mathcal{L}_{\mathcal{A}}(\mathcal{X})$, the unitary $U$ of the lemma above is of the form $U=e^{Z}$ for $Z \in \mathcal{L}_{\mathcal{A}}(\mathcal{X})$, with $Z^{*}=-Z$ and $\|Z\|<\pi / 3$ (using the same computation as in the norm of $I-V$ above).

Denote by

$$
\mathcal{L}_{x_{0}, x_{1}}=\left\{Z \in \mathcal{L}_{\mathcal{A}}(\mathcal{X}): Z^{*}=-Z, e^{Z}\left(x_{0}\right)=x_{1}\right\} .
$$

If $\left\|x_{0}-x_{1}\right\|<1 / 2$, then $\mathcal{L}_{x_{0}, x_{1}}$ is non empty. If $x_{0}, x_{1}$ are not that close, but they lie in the same component of $\mathcal{S}_{\mathcal{X}}$, the algebra $\mathcal{A}$ is a von Neumann algebra, and the module $\mathcal{X}$ is selfdual, one also has that $\mathcal{L}_{x_{0}, x_{1}}$ is non empty, with the unitary chosen such that $\|Z\| \leq \pi$. If moreover $\mathcal{A}$ is finite, then $\mathcal{S}(\mathcal{X})$ is connected, and any pair of elements in the sphere are conjugate by an exponential.

The following result is an adaptation of Theorem 3.2 in [8], to our particular context, where the Finsler metric is given by the norm of $\mathcal{X}$ (in [8] quotient norms are considered).

Theorem 4.3 Let $x_{0}, x_{1} \in \mathcal{S}_{\mathcal{X}}$, with $\left\|x_{0}-x_{1}\right\|<1 / 2$. Suppose that there exists $Z_{0} \in \mathcal{L}_{x_{0}, x_{1}}$ such that

$$
\left\|Z_{0}\right\|=\inf \left\{\|Z\|: Z \in \mathcal{L}_{x_{0}, x_{1}}\right\} .
$$

Then $Z_{0}$ is a minimal lift and therefore $\nu(t)=e^{t Z_{0}}\left(x_{0}\right)$ is minimizing along its path. In particular, it is shorter than any other piecewise smooth curve joining $x_{0}$ and $x_{1}$ in $\mathcal{S}_{\mathcal{X}}$.

Proof. The proof, as in 3.2 of [8], proceeds in three steps:

- a) Let $Z_{0} \in \mathcal{L}_{x_{0}, x_{1}}$ with $\left\|Z_{0}\right\|=\inf \left\{\|Z\|: Z \in \mathcal{L}_{x_{0}, x_{1}}\right\}$, fix $s \in(0,1)$ and denote $x_{s}=e^{s Z_{0}}\left(x_{0}\right)$. Then $s Z_{0} \in \mathcal{L}_{x_{0}, x_{s}}$ and $s\left\|Z_{0}\right\|=\inf \left\{\|Z\|: Z \in \mathcal{L}_{x_{0}, x_{s}}\right\}$. 
- b) Suppose that $X, Y$ are antihermitic operators of small norms in order that $e^{X} e^{Y}$ lies in the domain of the power series of the logarithm log defined on a neighbourhood of $I$ with antihermitic values. (for intance, $\left\|e^{X} e^{Y}-I\right\|<1$ ). Then

$$
\log \left(e^{X} e^{Y}\right)=X+Y+R_{2}(X, Y),
$$

where

$$
\lim _{s \rightarrow 0} \frac{R_{2}(s X, s Y)}{s}=0 .
$$

- c) Let $e=e_{x_{0}}$. For any $Y^{*}=-Y$ such that $Y=(I-e) Y(I-e)$, one has that

$$
\left\|Z_{0}\right\| \leq\left\|Z_{0}+Y\right\|
$$

Let us prove these steps, and show how they prove our result.

Step a):

For an element $X^{*}=-X$, denote by $\gamma_{X}(t)=e^{t X}$. We claim that the condition $\left\|Z_{0}\right\|=$ $\inf \left\{\|Z\|: Z \in \mathcal{L}_{x_{0}, x_{1}}\right\}$ implies that the curve $\gamma_{Z_{0}}$ is the shortest among piecewise smooth curves of unitaries joining $I$ to the set $\left\{U \in \mathcal{U}(\mathcal{H}): U\left(x_{0}\right)=x_{1}\right\}$. Indeed, by the remark above, since $\left\|x_{0}-x_{1}\right\|<1 / 2$, there exists $X \in \mathcal{L}_{x_{0}, x_{1}}$ such that $\|X\| \leq \pi / 3$. It follows that $\left\|Z_{0}\right\| \leq \pi / 3$. Suppose that $\mu(t)$ is another smooth curve of unitaries with $\mu(0)=I$ and $\mu(1)\left(x_{0}\right)=x_{1}$, which is shorter than $\gamma_{Z_{0}}$. Let $\mathcal{L}_{\mathcal{A}}(\mathcal{X})^{* *}$ be the von Neumann enveloping algebra of $\mathcal{L}_{\mathcal{A}}(\mathcal{X})$. Then there is a curve of the form $e^{t \Omega}, \Omega^{*}=-\Omega \in \mathcal{L}_{\mathcal{A}}(\mathcal{X})^{* *}$ and $\|\Omega\|<\pi / 3$, with $e^{\Omega}=\mu(1)$, which is shorter than $\mu$. This follows from the folklore fact that exponentials are short curves in the unitary group of a von Neumann algebra, when the length is measured with the Finsler metric given by the usual norm (see for instance [5]). It follows that $\|I-\mu(1)\|<3 / 2 . \mathrm{T}$

Let us show that $s\left\|Z_{0}\right\|=\inf \left\{\|Z\|: Z \in \mathcal{L}_{x_{0}, x_{s}}\right\}$. Suppose that there exists $X \in \mathcal{L}_{x_{0}, x_{s}}$ such that $\|X\|<s\left\|Z_{0}\right\|$. Consider the curve $\delta(t)=e^{(1-t) s Z_{0}+t Z_{0}}$ which joins $e^{s Z_{0}}$ with $e^{Z_{0}}$ in $\mathcal{U}(\mathcal{X})$, and $\sigma(t)=\delta(t) e^{-s Z_{0}} e^{X}$, joining $e^{X}$ and $e^{(1-s) Z_{0}} e^{X}$ (in both cases $t \in[0,1]$ ). Note that they have the same length, for they differ on an element of $\mathcal{U}(\mathcal{X})$ : length $(\delta)=$ length $(\sigma)=(1-s)\left\|Z_{0}\right\|$. Note also that the endpoint of $\sigma$ satisfies $\sigma(1) x_{0}=x_{1}$. Let $\tilde{\gamma}$ be the piecewise smooth curve which consists of the curve $\gamma_{X}$ followed by $\sigma$. Then $\tilde{\gamma}$ joins $I$ to the fiber $\left\{U \in \mathcal{U}(\mathcal{X}): U\left(x_{0}\right)=x_{1}\right\}$ in $\mathcal{U}(\mathcal{X})$, and therefore, by the fact remarked above, length $(\tilde{\gamma}) \geq\left\|Z_{0}\right\|$. On the other hand,

$$
\begin{gathered}
\operatorname{length}(\tilde{\gamma})=\text { length }\left(\gamma_{X}\right)+\text { length }(\sigma)=\|X\|+(1-s)\left\|Z_{0}\right\| \\
<s\left\|Z_{0}\right\|+(1-s)\left\|Z_{0}\right\|=\left\|Z_{0}\right\| .
\end{gathered}
$$

Step b):

The linear part of the series of $\log \left(e^{X} e^{Y}\right)$ is $X+Y$. So that

$$
\log \left(e^{X} e^{Y}\right)=X+Y+R_{2}(X, Y)
$$

Where the remainder term $R_{2}(X, Y)$ is an infinitesimal of the order $\|X\|+\|Y\|$. Therefore

$$
\lim _{s \rightarrow 0} \frac{R_{2}(s X, s Y)}{s}=0
$$

Step c):

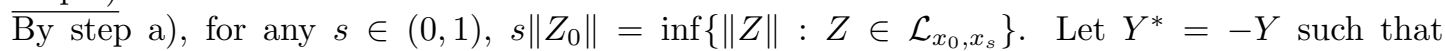
$Y=(I-e) Y(I-e)$. Then clearly $e^{Y}\left(x_{0}\right)=x_{0}$. Therefore $\log \left(e^{Z_{0}} e^{Y}\right) \in \mathcal{L}_{x_{0}, x_{1}}$. Analogously, $\log \left(e^{s Z_{0}} e^{s Y}\right) \in \mathcal{L}_{x_{0}, x_{s}}$. Then

$$
s\left\|Z_{0}\right\| \leq\left\|\log \left(e^{s Z_{0}} e^{s Y}\right)\right\|=\left\|s Z_{0}+s Y+R_{2}\left(s Z_{0}, s Y\right)\right\|
$$




$$
\leq s\left\|Z_{0}+Y\right\|+\left\|R_{2}\left(s Z_{0}, s Y\right)\right\|
$$

Then

$$
\left\|Z_{0}\right\| \leq\left\|Z_{0}+Y\right\|+\frac{\left\|R_{2}\left(s Z_{0}, s Y\right)\right\|}{s} .
$$

Taking limits, $\left\|Z_{0}\right\| \leq\left\|Z_{0}+Y\right\|$, which proves step c).

The theorem follows. The set $\left\{Z_{0}+Y: Y^{*}=-Y,(I-e) Y(I-e)=Y\right\}$ parametrizes the set of all $Z$ such that $Z e=Z_{0} e$. This means that $Z_{0}$ is a minimal lift, and therefore $\nu(t)=e^{t Z_{0}}\left(x_{0}\right)$ is a minimizing geodesic, joining $x_{0}$ and $x_{1}$.

Note that if $x_{0}, x_{1}$ are conjugate by the action of $\mathcal{U}(\mathcal{X})$, then the projections $e_{x_{0}}$ and $e_{x_{1}}$ are unitarily equivalent: if $U\left(x_{0}\right)=x_{1}, e_{x_{1}}=U\left(x_{0}\right) \otimes U\left(x_{0}\right)=U\left(x_{0} \otimes x_{0}\right) U^{*}=U e_{x_{0}} U^{*}$.

Corollary 4.4 Let $x_{0}, x_{1} \in \mathcal{S}_{\mathcal{X}}$, with $\left\|x_{0}-x_{1}\right\|<1 / 2$. Denote $f_{0}=1-e_{x_{0}}$. If the algebra $f_{0} \mathcal{L}_{\mathcal{A}}(\mathcal{X}) f_{0}$ is finite dimensional, then there exists a geodesic $\nu(t)=e^{t Z}\left(x_{0}\right)$ with $\nu(1)=x_{1}$, which is minimizing along its path.

Proof. Note that if $U, U^{\prime} \in \mathcal{U}(\mathcal{X})$ with $U\left(x_{0}\right)=U^{\prime}\left(x_{0}\right)$ it follows that $U^{*} U^{\prime}\left(x_{0}\right)=x_{0}$. Let $e_{0}=e_{x_{0}}$. This last statement is equivalent to $U^{*} U^{\prime} e_{0}=e_{0}$. The group $\mathcal{G}_{e_{0}}=\left\{V \in \mathcal{U}(\mathcal{X}): V e_{0}=e_{0}\right\}$ when written as $2 \times 2$ matrices in terms of $e_{0}$, consists of matrices of the form

$$
\left(\begin{array}{cc}
e_{0} & 0 \\
0 & f_{0} V f_{0}
\end{array}\right)
$$

where $f_{0} V f_{0}$ is a unitary operator in $\mathcal{U}\left(f_{0}(\mathcal{X})\right)$, which identifies with the unitary group of the reduced $C^{*}$-algebra $f_{0} \mathcal{L}_{\mathcal{A}}(\mathcal{X}) f_{0}$. It follows that $\mathcal{G}_{e_{0}}$ is compact in the norm topology. Therefore the set $\left\{U^{\prime} \in \mathcal{U}(\mathcal{X}): U^{\prime}\left(x_{0}\right)=x_{1}\right\}$ is compact, which implies that the set

$$
\left\{\|Z\|: Z \in \mathcal{L}_{x_{0}, x_{1}}\right\}
$$

has a minimum, and the theorem above applies.

Remark 4.5 If $\mathcal{A}$ is a von Neumann algebra and $\mathcal{X}$ is selfdual, then the hypothesis $\left\|x_{0}-x_{1}\right\|<$ $1 / 2$ of the above results can be replaced by the requirement that $x_{0}, x_{1}$ lie in the same connected component, or by no requirements at all if $\mathcal{A}$ is finite.

\section{$5 \quad$ Hilbert space spheres}

Denote by $d$ the metric in $\mathcal{S}_{\mathcal{X}}$ determined by the Finsler metric given by the norm of $\mathcal{X}$ at every tangent space of $\mathcal{S}_{\mathcal{X}}$ :

$$
d\left(x_{0}, x_{1}\right)=\inf \left\{\text { length }(\gamma): \gamma \text { joins } x_{0} \text { and } x_{1}\right\},
$$

with length $(\gamma)$ measured as before. As in the proof of the proposition (4.1) at the beginning of the preceding section, one may endow $\mathcal{X}$ with a semidefinite scalar product by means of a state $\psi$ of $\mathcal{A}$. Namely, put

$$
[x, y]_{\psi}=\psi(<x, y>), \quad x, y \in \mathcal{X} .
$$

If the state $\psi$ is non faithful this inner product degenerates. Let $\mathcal{Z}=\left\{z \in \mathcal{X}:[z, z]_{\psi}=0\right\}$ be the subspace of degenerate vectors, and $\mathcal{H}_{\psi}$ the completion of $\mathcal{X} / \mathcal{Z}$. Denote by $\bar{x}$ the class of $\in \mathcal{X}$ in $\mathcal{H}_{\psi}$. Note that the quotient map maps $\mathcal{S}_{\mathcal{X}}$ into $\mathcal{S}_{\mathcal{H}_{\psi}}$. If $x_{0}, x_{1} \in \mathcal{S}_{\mathcal{X}}$, denote by

$$
d_{\psi}\left(x_{0}, x_{1}\right)=\inf \left\{\text { length }(\alpha): \alpha \text { a smooth curve in } \mathcal{S}_{\mathcal{H}_{\psi}} \text { joining } \overline{x_{0}} \text { and } \overline{x_{1}}\right\},
$$


i.e. the geodesic distance of $\overline{x_{0}}$ and $\overline{x_{1}}$ as elements in the unit sphere $\mathcal{S}_{\mathcal{H}_{\psi}}$. Let

$$
d_{s}\left(x_{0}, x_{1}\right)=\sup \left\{d_{\psi}\left(x_{0}, x_{1}\right): \psi \text { a state in } \mathcal{A}\right\} .
$$

If $\left\|<x_{0}, x_{1}>\right\|<1$, a fact which implies that $\left[x_{0}, x_{1}\right]_{\psi}<1$, then it is a standard fact from the geometry of spheres (finite or infinite dimensional), that the distance equals

$$
d_{\psi}\left(x_{0}, x_{1}\right)=\arccos \left(\operatorname{Re}\left(\left[\overline{x_{0}}, \overline{x_{1}}\right]_{\psi}\right)\right)=\arccos \left(\operatorname{Re}\left(\psi\left(<x_{0}, x_{1}>\right)\right)\right) .
$$

Note that, for fixed elements $x_{0}, x_{1} \in \mathcal{S}_{\mathcal{X}}$, the map $\psi \mapsto \arccos \left(\operatorname{Re}\left(\psi\left(<x_{0}, x_{1}>\right)\right)\right)$ is continuous for the $w^{*}$-topology of the state space of $\mathcal{A}$. Therefore the supremum at the definition of $d_{s}$ is attained at a certain state. Note also that $d_{\psi}$ is in fact a pseudometric in $\mathcal{S}_{\mathcal{X}}$, if $\psi$ is not faithful.

Proposition $5.1 d_{s}$ is a metric in $\mathcal{S}_{\mathcal{X}}$. Moreover

$$
d_{s}\left(x_{0}, x_{1}\right) \leq d\left(x_{0}, x_{1}\right) .
$$

Proof. The metric $d_{s}$ is the supremum of a familiy of pseudometrics in $\mathcal{S}_{\mathcal{X}}$, therefore it is a pseudometric. Let us show that if $d_{s}\left(x_{0}, x_{1}\right)=0$ then $x_{0}=x_{1}$. Clearly this implies that $\overline{x_{0}}=\overline{x_{1}}$ in every Hilbert space $\mathcal{H}_{\psi}$, that is, $\psi\left(<x_{0}-x_{1}, x_{0}-x_{1}>\right)=0$ for all states $\psi$. This implies that $<x_{0}-x_{1}, x_{0}-x_{1}>=0$ and therefore $x_{0}=x_{1}$.

If $\gamma$ is a smooth curve in $\mathcal{S}_{\mathcal{X}}$ with $\gamma(0)=x_{0}$ and $\gamma(1)=x_{1}$, then

$$
[\dot{\bar{\gamma}}, \dot{\bar{\gamma}}]_{\psi}=\psi(<\dot{\gamma}, \dot{\gamma}>) \leq\|\dot{\gamma}\|^{2} \text {. }
$$

Next we show that these two metrics coincide if there exists a minimizing geodesic giving by a minimal lift as in the first section (Theorem 3.1). To prove this fact we need the following elementary results concerning states and operators in $\mathcal{L}_{\mathcal{A}}(\mathcal{X})$.

Lemma 5.2 Let $x_{0} \in \mathcal{S}_{\mathcal{X}}$ and $e=e_{x_{0}}$. Then $\mathcal{A}$ is isomorphic to the reduced algebra e $\mathcal{L}_{\mathcal{A}}(\mathcal{X})$ e, via the mapping $a \mapsto x_{0} a \otimes x_{0}$

Proof. The map $a \mapsto x_{0} a \otimes x_{0}$ is clearly linear, and takes values in $e \mathcal{L}_{\mathcal{X}}(\mathcal{A}) e: e\left(x_{0} a \otimes x_{0}\right) e=$ $x_{0} a \otimes x_{0}$. It is multiplicative:

$$
\left(x_{0} a \otimes x_{0}\right)\left(x_{0} b \otimes x_{0}\right)=x_{0} a<x_{0}, x_{0} b>\otimes x_{0}=x_{0} a b \otimes x_{0} .
$$

It preserves the adjoint: $\left(x_{0} a \otimes x_{0}\right)^{*}=x_{0} \otimes x_{0} a=x_{0} a^{*} \otimes x_{0}$. It is isometric: as remarked before, $\left\|x_{0} a \otimes x_{0}\right\|=\left\|x_{0} a\right\|\left\|x_{0}\right\|=\|a\|$. Finally, it is onto: if $T \in e \mathcal{L}_{\mathcal{A}}(\mathcal{X}) e$, then

$$
T=\left(x_{0} \otimes x_{0}\right) T\left(x_{0} \otimes x_{0}\right)=\left(x_{0} \otimes x_{0}\right)\left(T\left(x_{0}\right) \otimes x_{0}\right)=x_{0}<x_{0}, T\left(x_{0}\right)>\otimes x_{0},
$$

i.e. $T$ is the image of $<x_{0}, T\left(x_{0}\right)>\in \mathcal{A}$.

A straightforward consequence of this result is the following (see [4]).

Lemma 5.3 If $\Phi$ is a state of $\mathcal{L}_{\mathcal{A}}(\mathcal{X})$ with support less or equal than $e=x_{0} \otimes x_{0}$ (i.e. $\Phi(e)=1$ ), then there exists a state $\psi$ of $\mathcal{A}$ such that

$$
\Phi(T)=\psi\left(<x_{0}, T\left(x_{0}\right)>\right), \quad T \in \mathcal{L}_{\mathcal{A}}(\mathcal{X}) .
$$


Theorem 5.4 Let $x_{0}, x_{1} \in \mathcal{S}_{\mathcal{X}}$ with $\left.\|<x_{0}, x_{1}\right\rangle \|<1$, and suppose that there exists a minimal lift $Z$ at $x_{0}$ (i.e. $Z \in \mathcal{L}_{\mathcal{A}}(\mathcal{X}), Z^{*}=-Z$, with $\|Z\|=\|Z e\|=\left\|Z\left(x_{0}\right)\right\| \leq \pi$ ) such that $e^{Z}\left(x_{0}\right)=x_{1}$. Then the length of the geodesic $\nu(t)=e^{t Z}\left(x_{0}\right)$ equals the distance $d_{s}\left(x_{0}, x_{1}\right)$. In other words,

$$
d\left(x_{0}, x_{1}\right)=d_{s}\left(x_{0}, x_{1}\right)=\|Z\| .
$$

In particular, $\nu$ is a minimazing geodesic in $\mathcal{S}_{\mathcal{X}}$.

Proof. As in the proof of theorem 3.1, let $\xi$ be a norming (unit) eigenvector for $e Z^{2} e$ in a faithful representation of $\mathcal{L}_{\mathcal{A}}(\mathcal{X})$ : (as before we identify operators with their images under this representation) $e Z^{2} e \xi=-\|Z e\|^{2} \xi=-\|Z\|^{2} \xi$. Recall that $\xi$ lies in the range of $e$, and is also a norming eigenvector for $Z^{2}$. Consider the state $\Phi$ of $\mathcal{L}_{\mathcal{A}}(\mathcal{X})$ given by $\xi: \Phi(T)=[T \xi, \xi]_{\mathcal{H}}$ (here $[,]_{\mathcal{H}}$ denotes the inner product of $\mathcal{H})$. Then $\Phi(e)=1$, and therefore there exists a state $\varphi$ of $\mathcal{A}$ such that $\varphi(a)=\Phi\left(x_{0} a \otimes x_{0}\right)$. We claim that the state $\varphi$ realizes the maximum above:

$$
d_{s}\left(x_{0}, x_{1}\right)=\max \left\{\arccos \left(\operatorname{Re}\left(\psi\left(<x_{0}, x_{1}>\right)\right)\right): \psi \text { a state of } \mathcal{A}\right\} .
$$

To prove our claim, let us show that $\arccos \left(\operatorname{Re}\left(\varphi\left(<x_{0}, x_{1}>\right)\right)\right)=\|Z\|=d\left(x_{0}, x_{1}\right)$, which ends the proof. Note that

$$
\Phi\left(e^{Z}\right)=\Phi\left(\left(x_{0} \otimes x_{0}\right) e^{Z}\left(x_{0} \otimes x_{0}\right)\right)=\Phi\left(\left(x_{0}<x_{0}, e^{Z}\left(x_{0}\right)>\otimes x_{0}\right)=\varphi\left(<x_{0}, x_{1}>\right) .\right.
$$

On the other hand, $\Phi\left(e^{Z}\right)=\left[e^{Z} \xi, \xi\right]_{\mathcal{H}}$. Since $Z^{2} \xi=-\|Z\|^{2} \xi$, it follows that

$$
e^{Z} \xi=\left(1-\frac{1}{2}\|Z\|^{2}+\frac{1}{4 !}\|Z\|^{4}+\ldots\right) \xi+\left(1-\frac{1}{3 !}\|Z\|^{2}+\frac{1}{5 !}\|Z\|^{4}+\ldots\right) Z \xi .
$$

Note that since $Z$ is antihermitic, it follows that

$$
\operatorname{Re}\left(\left[e^{Z} \xi, \xi\right]_{\mathcal{H}}\right)=\cos \|Z\| .
$$

Therefore

$$
\operatorname{Re}\left(\varphi\left(<x_{0}, x_{1}>\right)\right)=\operatorname{Re}\left(\Phi\left(e^{Z}\right)\right)=\cos \|Z\| .
$$

It is a standard fact that given a state $\psi$ of $\mathcal{A}$, the algebra $\mathcal{L}_{\mathcal{A}}(\mathcal{X})$ can be represented in $\mathcal{H}_{\psi}$. Let us denote by $\rho_{\psi}$ this representation. Namely, if $x, y \in \mathcal{X}$ and $A \in \mathcal{L}_{\mathcal{A}}(\mathcal{X})$, then

$$
<A(x-y), A(x-y)>=<A^{*} A(x-y), x-y>\leq\|A\|^{2}<x-y, x-y>,
$$

therefore

$[A(x-y), A(x-y)]_{\psi}=\psi(<A(x-y), A(x-y)>) \leq\|A\|^{2} \psi(<x-y, x-y>)=\|A\|^{2}[x-y, x-y]_{\psi}$.

This implies that if $x$ and $y$ are equivalent in $\mathcal{X} / \mathcal{Z}$, then $A(x)$ and $A(y)$ are also equivalent, and the linear map $\bar{x} \mapsto A \overline{(x)}$ extends to a bounded operator $\rho_{\psi}(A)$ on $\mathcal{H}_{\psi}$.

Remark 5.5 Let $x_{0} \in \mathcal{S}_{\mathcal{X}}$ and $v \in\left(T \mathcal{S}_{\mathcal{X}}\right)_{x_{0}}$ with $\|v\| \leq \pi$. Suppose that there exists a minimal lift $Z \in \mathcal{L}_{\mathcal{A}}(\mathcal{X})$ for $v$. Let $\varphi$ be a state in $\mathcal{A}$ constructed as in the proof of the previous result. Then $\overline{x_{0}} \in \mathcal{H}_{\varphi}$ is an eigenvector for $\rho_{\varphi}\left(Z^{2}\right)$, with eigenvalue $-\|Z\|^{2}=-\|v\|^{2}$.

Let $Z$ be a minimal lift for $v$, i.e. $Z^{*}=-Z, Z\left(x_{0}\right)=v$ and $\|Z\|=\|v\|$. By Theorem (3.1), the curve $\nu(t)=e^{t Z} x_{0}$ has minimal length along its path in $\mathcal{S}_{\mathcal{X}}$. Then $\bar{\nu}$ is a minimizing geodesic in the Hilbert space sphere $\mathcal{S}_{\mathcal{H}_{\varphi}}$. Then $\ddot{\bar{\nu}}=-k^{2} \bar{\nu}$ for some real constant $k$. Therefore

$$
-k^{2} \bar{\nu}(t)=\ddot{\bar{\nu}}(t)=\rho_{\varphi}\left(Z^{2}\right) \bar{\nu}(t),
$$


i.e. $e^{t \rho_{\varphi}(Z)}\left(-k^{2} \overline{x_{0}}\right)=e^{t \rho_{\varphi}(Z)}\left(\rho_{\varphi}\left(Z^{2}\right)\left(\overline{x_{0}}\right)\right)$, which implies that

$$
\rho_{\varphi}\left(Z^{2}\right)\left(\overline{x_{0}}\right)=-k^{2} \overline{x_{0}} .
$$

On the other hand

$$
\left[\rho_{\varphi}\left(Z^{2}\right)\left(\overline{x_{0}}\right), \overline{x_{0}}\right]_{\varphi}=\varphi\left(<Z^{2}\left(x_{0}\right), x_{0}>\right)=\Phi\left(e Z^{2} e\right)=-\|Z\|^{2} .
$$

It follows that $k^{2}=\|Z\|^{2}$.

Combining the previous theorem with (4.4) one obtains the following:

Corollary 5.6 If the algebra $f_{0} \mathcal{L}_{\mathcal{X}}(\mathcal{A}) f_{0}$ is finite dimensional, and $x_{0}, x_{1}$ lie in the same connected component of $\mathcal{S}_{\mathcal{X}}$, then

$$
d\left(x_{0}, x_{1}\right)=d_{s}\left(x_{0}, x_{1}\right) .
$$

Proof. Note that $\left.\|<x_{0}, x_{1}\right\rangle \| \leq 1$. Suppose that $\left\|<x_{0}, x_{1}>\right\|<1$. By (4.4), there exists a minimal lift $Z \in \mathcal{L}_{\mathcal{A}}(\mathcal{X}), Z^{*}=-Z,\|Z\| \leq \pi$, such that $e^{Z}\left(x_{0}\right)=x_{1}$. Then the above theorem (5.4) applies and $d_{s}\left(x_{0}, x_{1}\right)=d\left(x_{0}, x_{1}\right)$. If $\left.\|<x_{0}, x_{1}\right\rangle \|=1$, then $x_{1}$ can be approximated by $x_{n} \in \mathcal{S}_{\mathcal{X}}$ (in the norm of $\mathcal{X}$ ), with $\left\|<x_{0}, x_{n}>\right\|<1$. It follows that $d_{s}\left(x_{0}, x_{n}\right)=d\left(x_{0}, x_{n}\right)$. Next note that if $\left\|x_{n}-x_{1}\right\| \rightarrow 0$, then $\left[\overline{x_{n}}-\overline{x_{1}}, \overline{x_{n}}-\overline{x_{1}}\right]_{\psi} \rightarrow 0$ for every state $\psi$. On the other hand also it is clear that $d\left(x_{n}, x_{1}\right) \rightarrow 0$. Therefore the result follows.

\section{References}

[1] Andruchow, E., Corach, G. Mbekhta, M. On the geometry of generalized inverses, Math. Nachr. 278 (2005), no. 7-8, 756-770.

[2] Andruchow, E., Corach, G., Stojanoff, D. Geometry of the sphere of a Hilbert module, Math. Proc. Cambridge Philos. Soc. 127 (1999), no. 2, 295-315.

[3] Andruchow, E., Corach, G., Stojanoff, D. Projective spaces of a $C^{*}$-algebra, Integ. Equat. Oper. Th. 37 (2000), no. 2, 143-168.

[4] Andruchow, E., Varela, A. $C^{*}$-modular vector states, Integr. Equ. Oper. Th. 52 (2005), 149-163.

[5] Atkin, C. J. The Finsler geometry of groups of isometries of Hilbert space. J. Austral. Math. Soc. Ser. A (1987), 196-222.

[6] Davis, C., Kahan, W. M., Weinberger, H. F. Norm preserving dilations and their applications to optimal error bounds. SIAM J. Numer. Anal. 19 (1982), 445-469.

[7] Durán, C. E., Mata-Lorenzo, L. E., Recht, L. Metric geometry in homogeneous spaces of the unitary group of a $C^{*}$-algebra. Part I. Minimal curves. Adv. Math. 184 (2004), no. $2,342-366$.

[8] Durán, C. E., Mata-Lorenzo, L. E., Recht, L. Metric geometry in homogeneous spaces of the unitary group of a $\mathrm{C}^{*}$-algebra. Part II. Geodesics joining fixed endpoints, preprint 310, Instituto Argentino de Matemática, CONICET (2003).

[9] Kobayashi, S., Nomizu, K. Foundations of differential geometry, Vol. II. Reprint of the 1969 original, Wiley Classics Library, John Wiley \& Sons, New York, 1996.

[10] Krein, M. G. The theory of selfadjoint extensions of semibounded Hermitian transformations and its applications. Mat. Sb. 20 (1947), 431-495; 21 (1947), 365-404 (in Russian). 
[11] Lance, E.C., Hilbert $\mathrm{C}^{*}$-modules, A toolkit for operator algebraists, London Math. Soc. Lecture Notes Series 210, Cambridge University Press, Cambridge, 1995.

[12] Halmos, P. R., McLaughlin, J. E. Partial isometries. Pacific J. Math. 13 (1963), 585596.

[13] Mata-Lorenzo, L.E., Recht, L. Infinite-dimensional homogeneous reductive spaces. Acta Cient. Venezolana 43 (1992), 76-90.

[14] Parrott, S., On a quotient norm and the Sz.-Nagy-Foias lifting theorem. J. Funct. Anal. 30 (1978), no. 3, 311-328.

[15] Paschke, W. L. Inner product modules over $B \mathcal{S}_{p}(\mathcal{A}) *$-algebras, Trans. Amer. Math. Soc. 182 (1973), 443-468

[16] Riesz, F., Sz.-Nagy, B. Functional Analysis. Ungar, New York, 1972

Esteban Andruchow and Alejandro Varela

Instituto de Ciencias

Universidad Nacional de Gral. Sarmiento

J. M. Gutierrez 1150

(1613) Los Polvorines

Argentina

e-mails: eandruch@ungs.edu.ar, avarela@ungs.edu.ar 